\title{
Bibliotecología para América Latina y el Caribe, propuesta teórica y filosófica para la discusión
}

\author{
Biblioteconomia para a América Latina e Caribe, proposta teórica e filosófica \\ para discussão \\ Librarianship from the southern cone, theoretical and philosophical proposal \\ for discussion
}

\author{
Natalia Duque-Cardona a \\ María Camila Restrepo-Fernández a,* (1)
}

\begin{abstract}
RESUMEN: La Bibliotecología emergió del seno de la práctica bibliotecaria, sus orígenes están signados por el desarrollo de sistemas organizativos para colecciones bibliográficas y la necesidad social de la existencia de bibliotecas públicas, hasta su constitución como disciplina científica, proceso caracterizado en una permanente obligación de renovación teórica y metodológica afín de responder a las cambiantes estructuras del quehacer científico y las tendencias globales de la tecnociencia. En este texto se presenta una propuesta teórica y filosófica que retoma las epistemologías del sur; primero, se evalúa el impacto e influencia del paradigma dominante de ciencia (realismo) en la bibliotecología; posteriormente, se revisan los fundamentos teóricos elementales: lenguaje, información, conocimiento, documento y biblioteca en clave sur y finalmente, se expone la propuesta de fundamentación filosófica para una bibliotecología desde Abya-Yalaa.
\end{abstract}

Palabras clave: Bibliotecología; Práctica Bibliotecaria; Epistemologías del Sur; Fundamentos de Bibliotecología.

RESUMO: A Biblioteconomia surgiu das práticas em biblioteca. Suas origens foram marcadas pelo desenvolvimento de sistemas de organização de coleções bibliográficas, e pela necessidade social da existência de bibliotecas públicas, até seu estabelecimento como disciplina científica, processo caracterizado por uma permanente obrigação de renovação teórica e metodológica, a fim de dar uma resposta às mudanças de estrutura do "o que fazer" científico e as tendências globais da tecnociência. Neste texto é apresentada uma proposta teórica e filosófica que retoma as epistemologias do sul. Primeiro se avalia o impacto e a influência do paradigma dominante da ciência (realismo) na biblioteconomia. Posteriormente, são revisados os fundamentos teóricos elementares: linguagem, informação, conhecimento, documento e biblioteca na chave do sul. Finalmente, se apresenta a proposta de fundamentação filosófica para uma biblioteconomia de Abya-Yala.

Palavras-chave: Biblioteconomia; Prática Bibliotecária; Epistemologias do Sul; Fundamentos da Biblioteconomía.

ABSTRACT: Librarianship is born the library practice, its origins are marked by the social need for the existence of public libraries and its transition to become a scientific discipline, it has been characterized by a permanent obligation

a Grupo de investigación Información, Conocimiento y Sociedad, Escuela Interamericana de Bibliotecología, Universidad de Antioquia, Medellín, Colombia.

* Correspondência para/Correspondence to: María Camila Restrepo-Fernández. Endereço: Universidad de Antioquia UdeA, Calle 70 No.52-21, Medellín, Colombia E-mail: mcamila.restrepo@udea.edu.co.

Recebido em/Received: 25/06/2021; Aprovado em/Approved: 27/11/2021.

Artigo publicado em acesso aberto sob licença CC BY 4.0 Internacional $@()$

a Abya-Yala es el nombre dado a lo que conocemos hoy como Latinoamérica en lengua del pueblo cuna. Este significa tierra en plena madurez o tierra de sangre. 
of theoretical and methodological renewal that responds to scientific changes and global trends in technoscience. This text presents a theoretical and philosophical proposal that takes up the epistemologies of the south. First, the impact and influence of the dominant paradigm of science (realism) in library science is evaluated. And then, the elementary theoretical foundations are reviewed: language, information, knowledge, document and library in a southern key. Finally, the proposal of philosophical foundation for a librarianship from Abya-Yala is exposed.

Keywords: Librarianship; Library Practice; Southern Epistemologies; Fundamentals of Library Science.

\section{INTRODUCCIÓN}

La bibliotecología objetivada en la biblioteca es una relación común validada por la historia del mundo y su afinamiento cultural, son variados los elementos presentes en la trasegar de las bibliotecas y la bibliotecología, la oralidad y la escritura, son quizás las más relevantes invenciones para lo que hoy conocemos e investigamos en materia de bibliotecas y bibliotecología, esta relación revela que los elementos mencionados son parte de lo que puede considerarse como el problema fundamental del pensamiento filosófico; el lenguaje.

El hecho de que por naturaleza los seres humanos se comunican es definitivo para comprender que la razón humana no se basa en la experiencia o pretende a la trascendencia como fin último y superior, sino que es la razón comunicativa el núcleo del ser humano en su sociedad. La historia de las bibliotecas denota con suficiencia y variados ejemplos el hecho mencionado, por ejemplo: la biblioteca de Alejandría pretendía reunir todos los libros existentes para llegar a ser la biblioteca universal, Alejandro, su creador, realizó magnánimas tareas para constituir una colección que incluyera todo lo que fuera conocido "fueron abolidas las fronteras, y allí convivieron, por fin en calma, las palabras de los griegos, los judíos, los egipcios, los iranios y los indios" (Vallejo, 2020, p. 25), en ese instante de la historia no se advertía aún el nacimiento posterior de la bibliotecología tal y como la conocemos, pero desde ese momento inicia su historia con una esencia eminentemente teórica de ordenación del conocimiento.

La historia de las bibliotecas, tema sobre el que abunda la literatura, no es objeto de este texto, más sí es un peldaño obligado para la bibliotecología, comprender que la disciplina bibliotecológica se pone en la escena científica del mundo es aceptar que las bibliotecas y su labor de organización, preservación y difusión del lenguaje, mayoritariamente escrito, han sido y se perpetúan como actividades intelectuales en el centro de la reflexión teórica y filosófica de la bibliotecología.

De modo que, el lenguaje encarnado en objetos documentales e informativos situados en un contexto histórico y social, son el objeto de estudio de la bibliotecología, esta debe proponerse desde una espacialidad concreta que ha de dar forma al uso y análisis de los objetos, sin embargo, debido a la interconexión global no optativa que vive el mundo, se proponen universales sobre los objetos y métodos para las disciplinas científicas, pero entendiendo que nada está establecido a priori y las verdades son fundamentalmente contextuales, revisitar la constitución científica de la bibliotecología a la luz del contexto propio, es decir, el latinoamericano y su

Liinc em Revista, Rio de Janeiro, v. 17, n. 2, e5727, nov. 2021. 
propuestas teórico-filosóficas, es hacer que las disciplinas científicas correspondan a su contexto, se erijan sobre la base de su utilidad social y perpetúen su desarrollo científico con sentido situacional.

Por ello, se revisa en este texto: el origen de la bibliotecología, fuertemente marcado por el pensamiento occidental desde sus inicios a la actualidad; la permanencia del pensamiento científico dominante en el desarrollo disciplinar de la bibliotecología; las propuestas alternativas de bibliotecología que han emergido en América Latina y El Caribe y la propuesta de análisis y discusión de las filosofías emergidas en La Región para la reformulación teórica y filosófica de una bibliotecología situada.

\section{ORIGEN DE LA BIBLIOTECOLOGÍA: UNA MIRADA DE OCCIDENTE AL SUR}

La sociedad ha determinado lo que ha sido la biblioteca del pasado, es la sociedad la que determinará lo que será la biblioteca del futuro.

Jesse Shera

La génesis de la bibliotecología va más allá de las discusiones actuales sobre su objeto de estudio, el germen de la bibliotecología es la necesidad de los individuos por comunicarse con otros individuos, por lo tanto, de usar el lenguaje en cualquiera de sus manifestaciones para hacer expresa la necesidad de comunicación. Así, la necesidad de comunicar información a través del lenguaje, en orden histórico: oral, escrito e impreso, es la base histórica en la que se asienta la bibliotecología.

Empero, una disciplina se asienta sobre múltiples bases, las que a su vez se forman y deforman como resultado del desarrollo humano y social, aunado al constante cambio tecnológico y la innovación científica, que, según la época, han tenido una mayor o menor implicación en la forma que llegan a tener las disciplinas. En el caso particular de la bibliotecología esta llega a constituirse como disciplina, en Occidente, debido a los requerimientos informativos necesarios para impulsar el desarrollo científico y tecnológico que dio como resultado el advenimiento y consolidación de la sociedad industrial a inicios del siglo XIX.

La industrialización de los procesos productivos y de la vida de los individuos, hizo urgente e impostergable cambiar la forma de educar a aquellos que integraban la fuerza de trabajo demandada para lograr el desarrollo acelerando la formación de los trabajadores, por lo que no bastó la escuela para responder a la urgencia de eficientar procesos educativos en pro del desarrollo industrial, sino que se requirió también de la biblioteca como institución mediadora entre los individuos trabajadores y el medio de trabajo.

Liinc em Revista, Rio de Janeiro, v. 17, n. 2, e5727, nov. 2021. 
De la mano de la necesidad educativa la producción de información registrada incrementó notablemente, con la fundación de Royal Society (1662) en Londres y su homóloga francesa Académie Royale des Sciences (1666), se inauguró la comunicación entre científicos a través de correspondencia, posteriormente, las mencionadas sociedades, iniciaron a divulgar sus avances mediante publicaciones periódicas, respectivamente: Philosophical transactions y Journal de Scavans. La práctica de publicar los avances y novedades científicas se extendió por todo occidente, siendo primero una práctica fuertemente adoptada por las ciencias médicas, exactas y naturales.

Sin embargo, fue sólo hasta el siglo XIX cuando las publicaciones seriadas de corte científico Ilegaron a su punto máximo alentadas por el desarrollo técnico, tecnológico y científico, de modo que la producción exacerbada información científica registrada exigía a las entidades responsables de su salvaguarda la renovación y creación de métodos de organización, almacenamiento y difusión de la información unívocos y estandarizados para simplificar y agilizar la recuperación de la información, elemento fundamental para la generación de nuevo conocimiento científico e impactar directamente en el progreso tecnológico.

Esta necesidad de mejorar los procesos de tratamiento de la información registrada reposa sobre la biblioteca y su personal, pues esta institución fue y continúa siendo, tanto en el imaginario colectivo como en la realidad social, incluso en diferentes contextos, el reservorio ideal para la información registrada. Así, la catalogación y clasificación comenzaron un progreso de tecnificación y estandarización vertiginoso, en forma cronológica se cuentan los siguientes hitos: Sistema clasificatorio de Brunet, 91 reglas de catalogación de Panizzi, Catálogo diccionario de Cutter, Clasificación Decimal de Dewey, Clasificación Decimal Universal de Otlet y la creación del Instituto Internacional de Bibliografía, son hechos son esenciales a la constitución de la bibliotecología como disciplina, sobre el sustento de que la correcta y rigurosa implementación de los procesos de organización eficienta la utilidad social de la información (Shera, 1976,1990).

Por lo tanto, los procedimientos organizacionales son fundacionales para la constitución de la bibliotecología como disciplina que inicia en el siglo XIX y se consolida en el siglo XX con la publicación de las primeras obras que le dan esta categoría, a saber: Tratado de documentación, y el inicio de la educación bibliotecológica en los claustros universitarios. La historia que tejió a lo largo del siglo XX para la bibliotecología estuvo marcada por un internacionalismo en favor la emisión de lineamientos y modelos de referencia para la organización e intercambio de información, especialmente información científica que era producida en los centros hegemónicos del conocimiento, a partir de la segunda mitad y hasta la finalización de la centuria el lanzamiento y continua actualización de normas como las ISBD (en todas sus versiones) y Anglo-American Cataloguing Rules, códigos como MARC21 y la automatización de los catálogos de biblioteca estuvieron dirigidas por las instituciones y asociaciones bibliotecarias predominantemente anglosajonas, teniendo un especial injerencia las de origen norteamericano. 
Dado que las normas, lineamientos y modelos para la organización e intercambio de la información fueron mantenidas y continúan siendo sostenidas por entidades de origen anglosajón y la participación de actores con origen diferente es baja, estas normas reflejan la visión de mundo propia de sus creadores, pesé a que se construyan sobre la base de la cooperación, al tratarse de herramientas que tecnifican el lenguaje para hacerlo transferible, unívoco y uniforme, terminan por siendo adecuaciones lingüísticas que eliminan la singularidad en nombre de la universidad como proyecto común.

Por otra parte, en lo referente al desarrollo de las formulaciones teóricas y filosóficas de la bibliotecología el camino recorrido no ha sido distinto al del quehacer bibliotecario, desde la profesionalización de la bibliotecología en las universidades anglosajonas hasta nuestros días, el relato teórico construido allí se ha constituido en la fuente primaria sobre la que se han construido y discuten los programas académicos de pregrado y posgrado en América Latina fortaleciendo así la continuidad de un paradigma dominante en bibliotecología, empero aún en este contexto se hace necesario que las formulaciones enunciadas hagan parte de los planes de estudio de los claustros universitarios donde se estudia la ciencia bibliotecológica, con el fin de situar a los estudiantes en una perspectiva histórica de esta que incluya el conocimiento de los teóricos clásicos e implique la presencia de referentes del Sur Global con el ánimo de reconocer, debatir e interpelar la ciencia en perspectiva histórica y dinámica. En el siguiente apartado, a la vez que se expone enfáticamente a los representantes de un pensamiento bibliotecológico situado se busca presentar un conocimiento situado alrededor que tensione los paradigmas dominantes y movilice la ciencia.

\section{LA BIBLIOTECOLOGÍA ATRAPADA POR EL PARADIGMA DOMINANTE: DE LA DOMINACIÓN A LA RESISTENCIA}

El paradigma dominante de la bibliotecología cuenta con representantes precisos debido a que sus esfuerzos por explicarla teórica y filosóficamente son ampliamente reconocidos, pues no abundan como en otras áreas del conocimiento, esto se explica en la juventud disciplinar de la bibliotecología. A continuación, se extractan los representantes, su afiliación institucional y los campos de desarrollo.

Tabla 1. Representantes del paradigma dominante en Bibliotecología.

\begin{tabular}{|l|l|l|}
\hline Representante & Afiliación institucional & Campos de desarrollo \\
\hline Shannon & $\begin{array}{l}\text { Instituto Tecnológico de } \\
\text { Massachusetts }\end{array}$ & $\begin{array}{l}\text { Teoría matemática de la } \\
\text { comunicación, enfoque } \\
\text { métrico de la información }\end{array}$ \\
\hline Landheer & Universidad de Groningen & $\begin{array}{l}\text { Enfoque sociológico a los } \\
\text { estudios sobre bibliotecas }\end{array}$ \\
\hline Borko & Universidad de California & Ciencia de la información \\
\hline Shera & Universidad de Chicago & Epistemología social \\
\hline Egan & Universidad de Chicago & \\
\hline
\end{tabular}




\begin{tabular}{|l|l|l|}
\hline Vichery & College of Londres & $\begin{array}{l}\text { Recuperación de la } \\
\text { información }\end{array}$ \\
\hline Hjorland & Royal School of Library and & Análisis de dominio \\
\cline { 1 - 1 } \cline { 3 - 3 } & Information Science & $\begin{array}{l}\text { Organización } \\
\text { conocimiento } \\
\text { Epistemología }\end{array}$ \\
\hline
\end{tabular}

Diseño propio

Los representantes explicitados en la Figura 1, hacen parte del corpus teórico sobre el que desarrolla una parte significativa de la investigación y enseñanza de la bibliotecología en América Latina. Cada uno de los autores mencionados se ubica al interior de centros universitarios pertenecientes a centro hegemónicos del conocimiento por lo que su lugar de enunciación del conocimiento obedece en forma directa a una concepción del mundo y la bibliotecología determinada por su contexto social, político y cultural privilegiado, si ve en relación con el contexto de América Latina y El Caribe.

Si bien la bibliotecología y la $\mathrm{Cl}$ se encuentran atrapadas aún por el paradigma dominante, con el advenimiento de pensamientos como la filosofía de la liberación (década de los 70) y la inflexión decolonial (década de los 90) en Latinoamérica y el Caribe hemos comenzado a observar en las Ciencias Humanas y Sociales, de las cuales hacen parte las ciencias en cuestión, una serie de procesos académicos y sociales de resistencia que van en perspectiva de desatarse de este paradigma dominante.

En relación con la filosofía de la liberación, su surgimiento en la década de los 70`permitió "pensar la colonialidad como punto de partida que permitió fenomenológicamente recuperar los símbolos del imaginario popular latinoamericano y políticamente intentar una militancia crítica no habitual en las filosofías meramente académica" (p.399), es a partir de esta que se comienzan procesos de reconfiguración de las ciencias y el hacer, que toman vida en contextos prácticos y académicos.

Si bien existen diversos procesos destacamos algunos casos de Colombia, Bolivia, Perú y México. Biblioghetto, es un colectivo colombiano que surge en Petecuy, barrio de la ciudad de Cali, Colombia, cuyo slogan es cambiando balas por libros. Un grupo de jóvenes con la firme convicción de que la leer y escribir son derechos y bienes comunes se fueron encontrando alrededor de la palabra, del lenguaje habilitaron un espacio abandonado y decidieron que este sería el inicio de un sueño que ya cumple 16 años

"Habilitaron una antigua caseta abandonada donde algunos drogadictos de la zona se reunían a meter bazuco, pusieron allí dos bibliotecas metálicas, cinco libros, dos carteleras, colgaron el nombre de un escritor para darse a sí mismos un poco de fuerza, y un buen día decidieron que ese sería el inicio del sueño, el pequeño rincón desde el cual empezarían la resistencia. A pocas cuadras está el basurero y varios recicladores viven colindando con los escombros. No hay con que pintar, no hay como ampliar la caseta, 
ninguna autoridad local ha querido unirse al proyecto y defender ese espacio mágico, ese cuadrado donde este combo de soñadores se reúne a leer con los chiquitos del sector." (Mendoza, 2018, p.7)

Biblioghetto y uno de sus fundadores Gustavo Andrés Gutiérrez, muestran como en la vida real, en el mundo de la vida, el derecho a la palabra, al lenguaje, humaniza y dignifica. Que siendo ciudadanos de a pie la lectura, la escritura y la oralidad, y para ellos el Grafiti que pinta las esquinas de lectura donde se reúnen con niños, niñas y jóvenes es un instrumento de poder, de resistencia y de vida.

En Medellín, Colombia, la segunda ciudad principal del país conocida mundialmente a causa de la memoria del narcotráfico, la cultura y las bibliotecas se empeñan y han logrado con trabajo colectivo y juntanza que su nombre se vincule al arte, la cultura y la vida, a las bibliotecas. Uno de los casos emblemáticos en este tema vinculado como una acción colectiva es Parque Al Barrio

"Esta iniciativa surge como una posibilidad después del cierre de la infraestructura del edificio del Parque Biblioteca Santo Domingo. El edificio funcionó durante siete años largos y en el 2015 se cierra definitivamente. Antes de ello se fueron realizando cierres paulatinos, de espacios, tratando de mantener vivo el funcionamiento al interior y batallando con lo que pasaba administrativamente en términos de la infraestructura y además con los medios de comunicación, pues estos influyeron de manera muy directa en el imaginario de la comunidad usuaria del parque, pues todo lo que nosotros tratábamos de defender y mantener abierto los medios lo destruyeron diciendo que el parque se iba a caer" (Duque, 2020, p.119)

En esta propuesta bibliotecaria, además de reconfigurar la idea de biblioteca como una infraestructura y proponer que el espacio bibliotecario trasciende las paredes de concreto, el trabajo comunitario y la vinculación con prácticas de educación popular través de pedagogía de la itinerancia han mantenido vivo un espacio bibliotecario emblemático para la ciudad es un acto de resistencia de la cultura. Natalia Espejo (2008), Coordinadora Técnica del Sistema de Bibliotecas Públicas de Medellín, relata que

"los habitantes de la zona que participan de las actividades consideran que la itinerancia estrecha los lazos entre los programas y su vida cotidiana. Un juego marcado por los lugares de cada jugador y por las reglas del mismo juego, donde tradicionalmente el bibliotecario es quien recibe en su espacio y bajo sus normas, al usuario que acude, lo transita y hace uso de él según lo institucionalmente establecido: no consumir alimentos dentro de la biblioteca, los animales de compañía deben quedarse fuera de las instalaciones, el horario es de 8:00 a.m. a 7:00 p.m., no montar los pies sobre las sillas, no sentarse en las mesas... tu turno. Ahora la biblioteca está en su casa, bajo sus reglas, ¿cómo sigue el juego? Se rompen los esquemas preestablecidos y con ello se reeditan los vínculos. Quien antes era el visitante ahora es el anfitrión y la biblioteca no está de visita, está de huésped." (p.91). 
Reconfigurar la idea de la biblioteca y lo que en esta acontece, así como las prácticas de promoción de la lectura es una tarea que acontece diariamente en diversos escenarios sociales, de hecho hay quienes además buscan que trascienda a generar un proceso de empoderamiento ciudadano que contribuya a dignificar la vida de las comunidades, como es el caso de la Biblioteca de la Creatividad, ubicada en la Vereda Quiba Guabal al sur de Bogotá, capital de Colombia. Esta es una iniciativa que tiene su génesis en el 2009 y es liderada por los bibliotecólogos Andrea Barón e Iván Triana

"Sus objetivos estratégicos se enfocan en crear un modelo de gestión que posicione las bibliotecas como huertos de ideas de emprendimiento, proyectos sociales e iniciativas para la paz, siendo la tecnología, la lectura y el juego las bases que dirigen su intervención comunitaria. Actualmente desarrolla tres proyectos institucionales orientados a fomentar el liderazgo, el emprendimiento, la innovación y la creatividad, como estrategia para disminuir la dependencia al asistencialismo en la comunidad." (Fundación Biblioseo, 2021)

Como vemos los procesos de reconfiguración y resistencia en el mundo de la vida se han centrado en la idea de la biblioteca y el lenguaje en específico, este último ha implicado una álgida discusión para la bibliotecología y la $\mathrm{Cl}$ que desjerarquice la cultura escrita como única forma de producción de conocimiento, en este campo una experiencia majestuosa acontece en Bolivia, con el Taller de tradición Oral Andina (THOA) fundado por Silvia Rivera Cusicanqui, el cual de manera consiente genera procesos de resistencia al colonialismo a través de la recuperación de la memoria que se ha salvaguardado gracias a la oralidad. Esta iniciativa data los 80 , momento en el que sus fundadores deciden contar la historia de los indios, gracias a esta iniciativa la memoria de algunos pueblos andinos se ha mantenido viva

"El Taller de Historia Oral Andina se crea el año 1983 alrededor de la Carrera de Sociología de la Universidad Mayor de San Andrés. Tras una reunión de trabajo en una casa particular de Sopocachi, los estudiantes de la materia de Superestructura Ideológica, que dictaba Silvia Rivera Cusicanqui, oficializaron su fundación a la cabeza de su docente. Para entrar en el grupo era un requisito el saber hablar un idioma nativo, sobre todo el aymara (...) Sin habérnoslo propuesto, habíamos ido desarrollando de una forma muy artesanal nuestra propia metodología de la historia oral basándonos en la memoria de los abuelos y abuelas." (Criales y Condoreno, 2016, p.61)

EI THOA además de desjerarquizar el castellano y dar prioridad a sus lenguas originarias (quechua y aymara) ha trabajado alrededor de diversas textualidades como: radionovelas, audiovisuales, entre otros, con más de tres décadas de trabajo en este ejercicio nos llamó a mirar los Andes, las filosofías indígenas y la potencialidad que estás bajo una mirada cuidadosa y amorosa nos posibilitan para salir de un paradigma dominante.

Finalmente, traemos a este espacio la Universidad de la Tierra que si bien no alude directamente a la biblioteca y la bibliotecología sí retoma preguntas fundamentales en su trabajo que para la

Liinc em Revista, Rio de Janeiro, v. 17, n. 2, e5727, nov. 2021. 
reconfiguración de las ciencias nos implican como disciplinas. Esta es una iniciativa que surge a partir del trabajo de comunidades Oaxaqueñas en el Foro Estatal Indígena de Oaxaca (2011) tras reconocer a la escuela como un instrumento de poder del Estado, lo cual no es lejano a la biblioteca la cual es un dispositivo colonial en tanto no se tramite su deconstrucción y en el marco de propuestas y pronunciamientos del Foro, se hace un llamado al

“el establecimiento de nuevas bases, principios, mecanismos e instituciones para hacer una revolución educativa en Oaxaca, que responda a nuestra realidad multiétnica y pluricultural, así como los estándares de calidad, y que sea un proceso democrático e incluyente. En especial se debe mejorar la calidad del servicio en las escuelas del nivel básico debiendo hacer realidad la educación indígena intercultural; el establecimiento de escuelas secundarias indígenas interculturales; el apoyo a los bachilleratos integrales comunitarios y la creación y apoyo de instituciones de educación superior en las regiones indígenas que sean acordes a sus realidades, culturas y lenguas." (Pueblos Indígenas de Oaxaca)

En Unitierra encontramos de modo particular el nosotros y el sentido comunal, el cual consideramos ineludible para los contextos bibliotecarios, esta propuesta retoma preguntas como ¿cómo crear acuerdos? ¿Cómo podemos construir, tomar y llevar a la práctica acuerdos colectivos? en esta iniciativa hay una insistencia en el bien común, en el nosotros, la cual es también la nuestra al ver la biblioteca como un bien común y la necesidad de que su trabajo nos convoque. Así como el llamado a espacios interculturales y saberes propios.

En la frontera Brasil, Paraguay, Argentina encontramos una iniciativa que presenta claramente la conformación de espacios bibliotecarios interculturales, el proyecto Vivendo libros latinoamericanos na Tríplice fronteira, que inició en el 2014 y es desarrollado por la UNILA. En este vemos que efectivamente la academia transita en procesos vinculados con la realidad que se distancian de los paradigmas dominantes. La fundadora de esta propuesta. Mariana Cortez (2017) insiste en la necesidad de propiciar "espacios de reemplazo de saberes y epistemologías, inclusión social, de refugio y principalmente de promoción de prácticas de lectura y de visibilización de las identidades que caracterizan la región (...) y así fundamentalmente reflexionar sobre la alteridad en el acervo bibliográfico" (p.40)

Las experiencias académicas que hemos propuesto se ubican en un paradigma otro y cómo revisamos tienen varias décadas de existencia, mientras que en el campo académico en Latinoamérica y el Caribe, desarrollos bajo un marco analítico y de interculturalidad crítica no superan las dos décadas. Hernando Lopera (2010), bibliotecólogo y actual director del Sistema de Bibliotecas de la Universidad de Antioquia, hace una década desarrolló su trabajo de investigación en maestría alrededor del giro decolonial, la interculturalidad y la diferencia colonial, su trabajo permitió el desarrollo de una pedagogía decolonial como alternativa de resistencia y reconstrucción de la sociedad, a partir de este generó un importante programa de formación en cibercultura para el contexto universitario.

Liinc em Revista, Rio de Janeiro, v. 17, n. 2, e5727, nov. 2021.

https://doi.org/10.18617/liinc.v17i2.5727 
EL concepto de bibliotecas interculturales, si bien ya había sido trabajado en contextos europeos en relación con el concepto de inmigración, en Latinoamérica comenzó a trabajarse con perspectiva diferencial e interseccional por Duque (2014) en su trabajo Entramado de voces: tejiendo bibliotecas interculturales en este mismo año también indaga acerca de las representaciones sociales de mujeres negras acerca de las LEO. Si bien hay trabajos anteriores respecto a bibliotecas indígenas estos recurren a perspectivas del paradigma dominante y se realizan bajo perspectivas multiculturales y no de interculturalidad crítica. En esta misma línea la investigadora desarrolla una propuesta teórica para la comprensión de la biblioteca como dispositivo cultural que vincula sus prácticas LEO con lenguajes políticos de la lectura, desarrollando en su trabajo el Lenguaje intercultural-decolonial de la lectura (2019). Como derivación de su ejercicio de fundamentación propone un "programa de acción/investigación de bibliotecas desde Abya-Yala: sociedades y culturas del Sur, compuesto por seis líneas temáticas que plantean una posible ruta, que más allá de concluir, permita vislumbrar acciones, caminos y estrategias para contribuir a la disminución de las desigualdades sociales en Latinoamérica" (2018, p.79).

En el contexto de la bibliotecología mexicana Felipe Meneses Tello, si bien no recurre a perspectivas interculturales e interseccionales desde los noventa presentaba un ejercicio vinculante con perspectivas críticas a la bibliotecología y en adelante sus análisis recurren a la sociología y la ciencia política para ampliar la comprensión de estas ciencias. Sus aportes han sido fundamentales para la constitución de un corpus teórico latinoamericano. Y Zapopan Martín Muela-Meza, ha tratado en su trabajo temáticas que al igual que en el caso de Meneses Tello retoman perspectivas críticas y alternativas para comprender la bibliotecología

Carolina Lema Flórez (2019), retoma desde la bibliotecología y la $\mathrm{Cl}$ el trabajo investigativo que vincula a pueblos originarios, indagando acerca de sus representaciones sociales acerca de la lectura, escritura, oralidad y biblioteca con sabedores y sabedoras Ẽbẽrã Chamí en contexto de ciudad para el tejido de saberes interculturales con la biblioteca pública.

Restrepo Fernández (2020) continúa en la línea de pensamiento latinoamericano en su investigación de maestría que aborda la alfabetización y la lectura como prácticas socioculturales para la inclusión social desde la biblioteca pública favoreciendo la inclusión de los individuos y grupos sociales en los procesos de participación políticos, culturales, económicos y educativos.

\section{LOS FUNDAMENTOS TEÓRICOS Y FILOSÓFICOS DE LA BIBLIOTECOLOGÍA EN CLAVE SUR: HILANDO EL ENTRAMADO}

Cimentar la bibliotecología y la Ciencia de la Información, nos impone retos importantes en clave de un Sur global, para lo cual las propuestas conceptuales que se desarrollan en este apartado parte de algunos horizontes de sentido propuestos por Fornet-Betacourt en sus disertaciones acerca de la filosofía intercultural (2009): 
- Las categorías, ideas aquí propuestas se liberan de un sistema único de saber occidentalizado y que ha privilegiado una tradición de los centros de poder epistémico como Europa y EE. UU.

- Reconocemos que el pensamiento filosófico occidental no es único, por lo cual una fundamentación en clave Sur requiere matrices culturales distintas y un diálogo que permita el intercambio de saberes

- Las discusiones y propuestas aquí planteadas no son universales, se contextualizan en América Latina y el Caribe, buscando alternativas que fundamenten la bibliotecología y la Ciencia de la Información en un territorio particular.

- Lo propuesto como fundamentos teóricos no es una propuesta para la academia, es una propuesta para tender puentes de una disciplina que se ha constituido bajo un modelo científico con las sociedades y la cultura en las cuales toma vida, " contribuyendo de esta manera a la formación de un espacio alternativo de opinión pública (p.643)”

- Discutir los fundamentos de la bibliotecología y la Ciencia de la Información está en la vía de ampliar el saber, el hacer y el sentido de estas ciencias, abriéndose a filosofías otras (campesinas, indígenas, andinas, feministas, interculturales, del Caribe), procurando unas ciencias construidas con los sujetos y no diseñadas para ellos.

Las propuestas teóricas latinoamericanas no son teorías totales, diferencia central en relación con las teorías occidentales de bibliotecología, pues estas tienen como propósito una integración totalitaria de los campos posibles en una única teoría, mientras que en los trabajos teóricos latinoamericanos la característica es la teorización en campos particulares y el desarrollo de programas de investigación en áreas bien delimitadas, por lo que se presume que afín de constituir una teoría refundacional de la bibliotecología desde América Latina y El Caribe, se deben revisitar las contribuciones hechas por los teóricos latinoamericanos y tejer una propuesta teórica común, sin pretensiones de universalidad.

En un nivel conceptual, la información como núcleo del trabajo bibliotecológico, cuenta con una propuesta: infodiversidad, concepto atribuido a Morales Campos (1998), investigadora mexicana que ha generado numerosos trabajos en esta línea de investigación proponiendo que la diversidad de la información se basa en la diversidad biológica y cultural que caracteriza a América Latina resultando en la infodiversidad "producto de la biodiversidad y diversidad cultural; es la gama del arcoíris que nos permite entender el todo y las partes, las semejanzas y diferencias entre regiones, entre bloques, entre grupos e individuos" (Morales Campos en Hernández Pérez, 2018, p. 31).

Lo relevante de la infodiversidad como parte de una teoría bibliotecológica desde el cono sur es que apuesta por la diversidad como una oportunidad de crecimiento de la información en base a la diversidad existente cultural y biológicamente, por lo que la recuperación y difusión de la diversidad es una tarea capital para las entidades oficiales, instituciones académicas y culturales, pues es el acceso a la información es un elemento central para el despliegue de la vida cotidiana de todos los ciudadanos en condiciones igualitarias, Hernández Pérez lo resume así "la 
información es hoy un elemento fundamental en el entramado social y productivo de nuestra sociedad; particularmente la información que producen culturas constituye un pilar fundamental en el desarrollo de la infodiversidad" (2018, p. 32).

Acuñando esta acepción de infodiversidad como núcleo de una teoría bibliotecológica en clave sur, se establece a la diversidad como un rasgo fundante y decisivo en la aplicación teórica, es decir, en el quehacer bibliotecológico, acentuando por ejemplo en la necesidad y obligación de reestablecer las prácticas de promoción de la oralidad, lectura y escritura en la diversidad de lenguas existentes en La Región, prácticas que se sitúan históricamente en instituciones como la escuela y la biblioteca, debido a los intereses y objetivos teóricos de este texto, se propone a la biblioteca como la institución desde la que se sitúan estas prácticas cuyo centro es la infodiversidad expresa la oralidad, lectura y escritura como prácticas socioculturales.

Por lo tanto, la biblioteca, es el segundo elemento conceptual que debe ser rediseñado en favor de una teoría bibliotecológica en clave cono sur. Las nociones de biblioteca abundan, existen tantas definiciones posibles como seres en el mundo, esto se explica en la multiplicidad de tipos de bibliotecas con las que contamos: biblioteca escolar, biblioteca pública, biblioteca universitaria, biblioteca especializada, entre otras. Sin embargo, para una propuesta teórica como la que aquí proponemos, fundamentada en la diversidad, es coherente tomar y ajustar propuestas conceptuales como biblioteca humana.

La biblioteca humana es una iniciativa que ha tenido gran acogida en América Latina, pues se trata de una práctica en la que los relatos de las personas son transmitidos a otras personas mediante el diálogo en las bibliotecas, en esta dialéctica, las personas que exteriorizan sus experiencias a través de la palabra hablada son consideradas como libros humanos, porque cumplen con característica de ser las depositarias del conocimiento que está siendo transferido. En esta mediación del lenguaje, también se incluye material documental como libros, revistas y audiovisuales, más no es definitivo,

"la Biblioteca Humana no es sólo una colección de libros, es principalmente una comunidad de aprendizaje, un laboratorio donde nos experimentamos juntos para abrazar nuestra complejidad. Una comunidad como la que fue necesaria en las bibliotecas de las abadías medievales para tener acceso a la escasa información. Una comunidad como la que paradójicamente se necesita también ahora que la información abunda, y nos necesitamos para dar sentido al mundo complejo que habitamos" (Epstein Cal y Mayor, 2019, p. 8)

En suma, la infodiversidad como el concepto germen, la biblioteca humana como la tipología bibliotecaria que enmarca y sitúa el quehacer bibliotecológica y la oralidad, lectura y escritura como prácticas materiales sobre las que trabaja la bibliotecología, conforman el objeto de estudio de la bibliotecología en clave sur cuyo presupuesto filosófico recae en las filosofías anticoloniales enmarcadas en lo que hoy se conoce como epistemologías del Sur, como una 
oportunidad y una deuda que comienza a saldarse con la historia propia de la bibliotecología latinoamericana y caribeña.

\section{ALTERNATIVAS FILOSÓFICAS PARA LA BIBLIOTECOLOGÍA LATINOAMERICANA Y DEL CARIBE DESDE LAS EPISTEMOLOGÍAS DEL SUR}

Cuando la tierra no era, en medio de las tinieblas primigenias, cuando no había conocimiento de las cosas, hizo florecer en sí el fundamento de la palabra; lo convirtió el verdadero Primer Padre Ñamandú en propia sabiduría divina.

Las epistemologías del Sur no son recientes, si bien este término es acuñado por De Sousa Santos las epistemes del Sur, de Abya-Yala, y pensamientos ancestrales anteceden 1492. Pensar que partimos de allí es un acto de completa arrogancia que sigue ubicada en una perspectiva dominante de la ciencia, por ello partimos de filosofías de algunos pueblos originarios tratando de hilar su saber son el conocimiento occidental, empero no nos limitamos a ellas. Retomamos también la filosofía del Caribe, la intercultural, la feminista, la de la liberación. Nos ubicamos en una perspectiva anticolonial que si bien reconoce a la universidad como una institución productora de conocimiento no se limita a ella.

Esta propuesta filosófica para la bibliotecología no busca adornar el lenguaje, poner en otras palabras "más interculturales, más originarias", lo que se quiere es tender puentes, pasar de reconocer a conocer cómo se camina la palabra y a redistribuir el conocimiento, el saber en una perspectiva de justicia social donde no sólo se privilegien los paradigmas dominantes de la ciencia. Por lo tanto, las alternativas filosóficas contextualizadas al territorio en perspectiva Sur, tiene implicaciones que van más allá de recurrir a los desarrollos teóricos producidos en la transmodernidad respecto a las epistemologías del Sur, pues incluso aquí la tarea es no sólo de reconocimiento sino de redistribución de saberes alrededor de diversas epistemes.

En este apartado retomamos algunas filosofías del pensamiento latinoamericano, del Caribe y "latino" en las cuales encontramos alternativas para "desfilosofar" la bibliotecología y la Ciencia de la Información, es decir para liberarlas "de la cárcel en la que todavía se mantienen prisioneras de la vigente hegemonía de la tradición occidental centroeuropea” (Fornet-Betancourt, 2009 p.642). Retomamos así algunas ideas de filosofías indígenas (náhuatl, maya, tojolabal, quechua, Mapuche, guaraní), del Caribe, feministas, libertarias e interculturales para presentarle a la bibliotecología y la Ciencia de la Información alternativas para caminar la palabra. 


\section{Tendiendo puentes, del pensamiento filosófico de Abya-Yala a la bibliotecología y la Ciencia de la Información}

\section{Filosofías indígenas}

Los sabios de los pueblos que hablaban lengua náhuatl se conocían como tlamatinime, "los que saben algo" "En lengua náhuatl neltiliztli, es término derivado del mismo radical que tlanél-huatl, raíz, del que a su vez directamente se deriva nelhuáyotl, cimiento, fundamento. La sílaba temática nel- connota originalmente la idea de enraizamiento profundo". En relación con esto, puede decirse que etimológicamente "verdad" entre los nahuas era, en su forma abstracta (neltiliztli), la cualidad de estar firme, bien cimentado o enraizado: lo fundado" (León-Portilla, 2009, p.24). Esta idea de cimientos es precisa para la discusión que venimos desarrollando, pues en el pensamiento maya encontramos para las ciencias en cuestión un llamado a un enraizamiento profundo que considere nuestras raíces y memoria, es decir que no parta solo del magno proyecto de Alejandro, sino que reconozca en su génesis procesos juiciosos alrededor del registro de la información, de la circulación de la palabra que ya existían mucho antes de las civilizaciones antiguas de occidente. El pensamiento náhualt permite en este caminar la palabra y de fundamentación del conocimiento reconocer que el camino por la verdad implica estar firmes y cimentados en lo que ha sido nuestra constitución como ciencias, para lo cual nos urge una historia de la bibliotecología, de la lectura desde el Sur.

Continuando con el pensamiento filosófico en lo que hoy conocemos como Centroamérica, la filosofía maya que retoma Hernández-Díaz (2009) parte de reconocer en uno de los objetos simbólicos más relevantes para la bibliotecología y la Ciencia de la Información: los libros, la noción de comunidad. "El Popol-vuh, libro sagrado, es un libro de la comunidad. El término maya Popol tiene el sentido de junta, reunión o casa común. Mientras que la palabra vuh o uúh significa libro, papel o trapo y se deriva del maya húun o úun; refiere al papel y al libro, y asimismo al árbol de cuya corteza se hacía el papel para escribir, y que los nahuas llaman amatl” (p.26). Vemos en el origen de esta palabra un sentido a lo comunitario, a lo comunal, a nosotros, nosotras, el cual es hoy una idea fundamental para el desarrollo bibliotecológico en Latinoamérica y El Caribe. “El Popol na es la casa de la comunidad, pero no simplemente es la casa para dialogar, sino que tiene mayor función como la casa grande donde se tratan los asuntos sobre diversos temas de la cultura" (p.26) ¿Y qué son nuestras bibliotecas sin la comunidad? El nosotros, lo comunitario, lo comunal es la savia que permite identificar las bibliotecas y la información como un bien común. La idea de juntanza como punto de partida da fuerza al quehacer bibliotecológico, tan fuertemente ligada a la labor que cumplen las bibliotecas y sus profesionales como promotores de la interacción social bajo la mediación de la transferencia de la información, recreando las funciones sociales de la bibliotecología en las comunidades y actores en quienes incide.

La filosofía Tojolabal (Lenkersdorf, 2009), de los altos de Chiapas, coincide con la maya al hacer énfasis en el nosotros, nosotros significa hablar de comunidad y la priorización del encuentro, en esta interacción la oralidad cobra un alto valor, pese a ser un elemento fundante del lenguaje es una de las categorías conceptuales que ha estado subordinada en la bibliotecología, debido a la 
predominancia de la cultura escrita como centro del estudio, como una alternativa que nos posibilite ciencias contextuales y consonantes con la realidad de Latinoamérica y el Caribe, es imperante que la oralidad tome el lugar de hermana mayor de la escritura y la lectura, y en tanto no sólo pase a ser parte constitutiva de los conceptos fundamentales de estas, sino que también nos provea la posibilidad de un diálogo de saberes con pueblos y saberes cuya información no ha privilegiado el código escrito.

Y caminando un poco más al Sur, encontramos la filosofía quechua (Estermann, 2009), la que retoma filosofías indígenas andinas de las cuales para este ejercicio retomamos las ideas de correspondencia y reciprocidad, ambos tienen un rol fundamental en este pensamiento y en el campo de la bibliotecología y la $\mathrm{Cl}$, nos hacen un llamado a tener presente las funciones sociales de la biblioteca (culturales, políticas, educativas y económicas) en las cuales radica la esencia del hacer bibliotecario.

El pensamiento Mapuche (Salas Astraín, 2009), en el campo de las filosofías del Sur nos permite como ciencias una pausa para pensar en una bibliotecología situada y contextualizada, en una biblioteca en relación con el mundo social. "El mapuche rakiduam entiende el pensamiento como brotando siempre desde el mundo de la vida (mapuche mogen) y asociado a normas y valores contextuales (admapu) específicos que afirman un modo de ser, un saber y un valorar que resiste a los procesos negadores de otros saberes hegemónicos" (p.42). Y es en ese mundo de la vida, en el cual la bibliotecología y la $\mathrm{Cl}$ se resisten a continuar siendo ciencias coloniales y la biblioteca a ser un dispositivo colonial, hoy la biblioteca y la bibliotecología trabajan arduamente para conseguir procesos situados y responder a las necesidades contextuales de Latinoamérica y el Caribe que implican miradas interculturales: el reconocimiento de la diversidad de sujetos, lenguas, lenguajes, textualidades.

La filosofía mapuche, destaca el concepto de comunidad en relación con la memoria y el lenguaje, pues para poder trabajar en una ciencia y hacer situado se requiere la recuperación de la memoria cultural que fue saqueada desde 1492 “ (...) no hay devenir de la comunidad sin referencia a una afirmación de la memoria ancestral” (p.43), este tipo de memoria ha estado ausente de los espacios bibliotecarios y de la bibliotecología, pues en términos de las matrices de conocimiento se han privilegiado las blancas, masculinas y europeas, empero la mirada a través de las epistemologías del Sur puede proveernos opciones para tener antiguas formas de conocimiento en nuevas formas de comprender la biblioteca y la bibliotecología. Incluso, el trabajo alrededor de la información local y la recuperación de saberes propios para la producción de conocimiento situado es un ejercicio en el que la filosofía mapuche insiste, este lo vincula con "una reconstrucción del saber centrada en el vínculo humano (che) a la tierra (mapu), que articula y sistematiza sus propios conocimientos (Kimün) que los producen y reproducen como parte de un saber cultural” (p.44), una alternativa para hacer de la biblioteca un centro de pensamiento propio.

Ahondar en las filosofías indígenas para meditar cómo caminar la palabra en esta Abya-Yala desde el campo de la bibliotecología y la $\mathrm{Cl}$, sitúa a la palabra como una tecnología de poder que 
ha sido usada como refugio, empero también como artefacto de saqueo. En relación con la palabra, la filosofía guaraní tiene una bella forma de referirse a esto que nombramos como caminar la palabra: "hacer florecer en sí la palabra oguerojerá”, "para el guaraní, la palabra lo es todo y todo para él es la palabra (...) el hombre al nacer será una palabra que se pone de pie y se yergue hasta su estatura plenamente humana" (Melià, 2009, p.49). La filosofía guaraní nos ofrece la oportunidad de una filosofía sapiencial de la palabra, de enaltecer la humana condición que tenemos a través del lenguaje que acontece en el campo de la vida, en espacios como las bibliotecas que están habitadas por la palabra, en ellas a diario entre muchos otros propósitos intentamos que florezca la palabra de otros, de otras, y por supuesto bajo un sentido comunal la nuestra.

\section{Filosofías del Caribe, feministas y de la liberación}

En el tránsito a la transmodernidad, además de las filosofías que se mueven alrededor del reconocimiento y producción de saber que visibiliza categorías étnico-raciales están también las que vinculan el género, y en específico el feminismo filosófico que puede proveer a la bibliotecología y $\mathrm{Cl}$ importantes insumos. Plantea Gargallo Celanti (2019) que "de hecho es el feminismo la primera filosofía que toma conciencia de las políticas de legitimidad, es decir, de las formas con que una sociedad otorga el privilegio cultural de legitimar los saberes y valores a un grupo (los hombres, los vencedores, los blancos, los aristócratas, los ricos) con el fin de excluir los aportes, saberes, valores, conocimientos de otros grupos (las mujeres, las esclavas, las pobres, las indígenas, las negras)" (p.420), a través del feminismo filosófico encontramos alternativas para nombrar, para aludir al respeto a las diferencias, para caminar hacia procesos que impliquen no sólo el reconocimiento sino también la redistribución. Como ciencias construidas bajo un paradigma dominante, los principios e ideas del feminismo nos permiten preguntarnos desde qué lugar hemos construido la historia de la bibliotecología, qué y a quiénes hemos dejado fuera de esta.

Finalmente, como filosofía de cierre a estas alternativas epistemológicas para la bibliotecología y la $\mathrm{Cl}$, está la filosofía de la liberación (Dussel, 2009), la cual consolida los conceptos que aportan las filosofías expuestas anteriormente y enfatiza en la necesidad urgente de una descolonización epistémica de la ciencia occidental para lograr que está tenga un compromiso con la praxis, bajo una perspectiva comunitaria, aludiendo al nosotros. Esta filosofía nos permite reconocer que en las disciplinas en cuestión se presentan bajo esquemas dominantes debido a procesos de colonialidad que perviven a través del lenguaje, la memoria y la información, categorías fundamentales para la bibliotecología. Así que como ya lo hemos mencionado estos pensamientos permiten desfilosofar estas ciencias y aperturarlas en perspectiva Sur. 
Tabla 2. Filosofías vs aportes conceptuales a la bibliotecología y la Cl

\begin{tabular}{|l|l|}
\hline Filosofía & \multicolumn{1}{|c|}{ Conceptos o ideas que aporta a la bibliotecología y la Cl } \\
\hline Náhuatl & Verdad (estar firmes), fundamentos (enraizamiento profundo) \\
\hline Maya & Comunitarismo, Comunalidad \\
\hline Tojolabal & Lenguas oídas, oralidad, comunitarismo, otredad \\
\hline Quechua & Funciones sociales de la biblioteca, correspondencia, reciprocidad \\
\hline Mapuche & Información local, memoria cultural, conocimiento situado, comunitarismo \\
\hline Guaraní & Lenguaje, humanidad, oralidad \\
\hline Del Caribe & Ruptura con cánones, oralidad, emancipación \\
\hline Feminismo & Reconocimiento, redistribución, equidad, inclusión, la fuerza de nombrar \\
\hline Liberación & $\begin{array}{l}\text { Decolonización del saber, compromiso con la praxis de una comunidad popular, } \\
\text { Comunitarismo }\end{array}$ \\
\hline
\end{tabular}

Diseño propio

\section{DISCUSIÓN. EPISTEMOLOGÍAS DEL SUR, BIBLIOTECOLOGÍA Y CI}

Las epistemologías del Sur son profundamente históricas, pero parten de otras historias que no son precisamente la historia universal de occidente

Boaventura De Sousa Santos

Irrumpir las formulaciones teóricas y filosóficas aceptadas hasta ahora como básicas y clásicas en la bibliotecología y la ciencia de la información y construir una nueva y coherente formulación teórica y filosófica desde y para América Latina y el Caribe, reivindicando el poder del conocimiento situado es una apuesta afirmativa asentada en las siguientes premisas:

1. La comprensión del mundo es mucho más amplia que la comprensión de occidente del mundo;

2. la diversidad del mundo es infinita $y$,

3. Esta gran diversidad del mundo puede y deber ser activada, así como transformada teórica y prácticamente de muchas maneras plurales, no puede ser monopolizada por una teoría general (Boaventura, 2018)

Las corrientes filosóficas indigenistas, feministas y decoloniales sumarizadas en el texto en lectura, aportan el dinamismo requerido para un marco filosófico capaz de 
comprender integralmente la diversidad del mundo más allá de occidente como centro hegemónico del conocimiento. Integrar estas corrientes al pensamiento de la Bibliotecología y $\mathrm{Cl}$ es tanto una innovación, como una deuda disciplinar de sus teóricas y teóricos, esta reflexión contribuirá al reconocimiento, redistribución y representación de los conocimientos, saberes y prácticas de América Latina y El Caribe.

El énfasis de las filosofías seleccionadas tiene como rasgo común la posición protagónica del lenguaje, en las que su valor instrumental sirve para salvaguarda sus cosmovisiones sobre el mundo, este es el motivo de selección, pues la comprensión filosófica de la Bibliotecología y CI desde América Latina y El Caribe se propone aquí como: una disciplina que reflexiona y trabaja sobre las distintas expresiones del lenguaje humano, especialmente el registrado y organizado, a la vez que reconoce en la oralidad una posibilidad para el progreso disciplinar teórico y un aumento en el alcance de la práctica profesional entre las comunidades del sur global.

Este marco filosófico requiere de la discusión profunda entre la comunidad científica y académica de América Latina, se propone un debate amplio en el que el propósito no sea elegir una filosofía de las enunciadas sino la integración filosófica, afín de proveer diferentes marcos de investigación para el conjunto de conceptos definidos como centro de análisis de la bibliotecología: infodiversidad, biblioteca humana y la oralidad, lectura y escritura.

En tanto se hace ineludible avanzar en la conformación de una propuesta de fundamentación que permita una mirada amplia, sin embargo, situada, de la bibliotecología y la ciencia de la información reconociendo la pluralidad del territorio en el campo simbólico de la diferencia. Una propuesta filosófica para la discusión de este tipo implica no solo poner en tensión la ciencia y el conocimiento científico hegemónico, sino incluso recurrir a métodos contra inductivos que permitan un verdadero desarrollo de la ciencia.

\section{REFERENCIAS}

CRIALES, Lucila ; CONDORENO, Cristobal, 2016. Breve reseña del Taller de Historia Oral Andina (THOA). Fuentes, 10, no. 43, p. 57-66. [Acceso 21 de noviembre de 2021] Disponible en http://www.revistasbolivianas.org.bo/pdf/fdc/v10n43/v10n43_a12.pdf

CORTEZ, Mariana; GORDILLO, Daniel, 2017. A leitura em contexto de fronteira: Acervos e salas de leitura interculturais. En: DE SOUSA, Ivan, Leitura na Educação Básica: perspectivas e desafios. Jundiaí: Paco Editorial, p. 29-46.

DE SOUSA SANTOS, Boaventura, 2018. Construindo as epistemologias do Sul: Antologia essencial. Volume I: para um pensamento alternativo de alternativas. Buenos Aires: CLACSO.

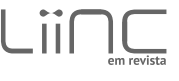

Liinc em Revista, Rio de Janeiro, v. 17, n. 2, e5727, nov. 2021.

https://doi.org/10.18617/liinc.v17i2.5727 
DUQUE, Natalia, 2014. Tejiendo Bibliotecas Interculturales. Medellín : Fundación Taller de Letras Jordi Sierra i Fabra, Alcaldía de Medellín. Secretaría de Cultura Ciudadana.

DUSSEL, Enrique; MENDIETA, Eduardo; BOHÓRQUEZ, Carmen, 2009. El pensamiento filosófico latinoamericano, del Caribe y "latino" (1300-2000) : historia, corrientes, temas y filósofos. México : Siglo XXI: Centro de Cooperación Regional para la Educación de Adultos en América Latina y el Caribe.

EPSTEIN CAL Y MAYOR, Lourdes, 2019. Cómo hacer una biblioteca humana. España: Ministerio de Educación.

ESPEJO, Natalia, 2018. Parque al Barrio: la biblioteca itinerante de Medellín (Colombia). Mi biblioteca, XIV, 53, p.88-93.

HERNÁNDEZ DÍAZ, Miguel, 2009. La filosofía Maya. En: MENDIETA, Eduardo; BOHÓRQUEZ, Carmen. El pensamiento filosófico latinoamericano, del Caribe y "latino" (1300-2000) : historia, corrientes, temas y filósofos. México : Siglo XXI: Centro de Cooperación Regional para la Educación de Adultos en América Latina y el Caribe. p. 2733.

HERNÁNDEZ PÉREZ, Jonathan, 2018. Infodiversidad en internet. México : Universidad Nacional Autónoma de México.

LENKERSDORF, Carlos, 2009. La filosofía Tojolabal. En: MENDIETA, Eduardo; BOHÓRQUEZ, Carmen. El pensamiento filosófico latinoamericano, del Caribe y "latino" (1300-2000) : historia, corrientes, temas y filósofos. México : Siglo XXI: Centro de Cooperación Regional para la Educación de Adultos en América Latina y el Caribe. p. 3335.

GARGALLO CELANTI, Francesca, 2009. El feminismo filosófico. En: MENDIETA, Eduardo; BOHÓRQUEZ, Carmen. El pensamiento filosófico latinoamericano, del Caribe y "latino" (1300-2000) : historia, corrientes, temas y filósofos. México : Siglo XXI: Centro de Cooperación Regional para la Educación de Adultos en América Latina y el Caribe. p. 418433.

ESTERMANN, Josef, 2009. La filosofía quechua. En: MENDIETA, Eduardo; BOHÓRQUEZ, Carmen. El pensamiento filosófico latinoamericano, del Caribe y "latino" (1300-2000) : historia, corrientes, temas y filósofos. México : Siglo XXI: Centro de Cooperación Regional para la Educación de Adultos en América Latina y el Caribe. p. 33-41.

FORNET-BETANCOURT, Raúl, 2009. La filosofía Intercultural. En: MENDIETA, Eduardo; BOHÓRQUEZ, Carmen. El pensamiento filosófico latinoamericano, del Caribe y "latino" (1300-2000) : historia, corrientes, temas y filósofos. México : Siglo XXI: Centro de Cooperación Regional para la Educación de Adultos en América Latina y el Caribe. p. 639646. 
FUNDACIÓN BIBLIOSEO. (2021). Biblioteca de la creatividad. [Acceso 21 de noviembre de 2021] Disponible en https://biblioseo.org/about-us/

LANDHEER, Bartholomeus, 1957. Social functions of libraries. New York: Scarecrow.

MELIÁ, Bartomeu, 2009. La filosofía guaraní. En: Mendieta, E. y Bohórquez, C. El pensamiento filosófico latinoamericano, del Caribe y "latino" (1300-2000) : historia, corrientes, temas y filósofos. México : Siglo XXI: Centro de Cooperación Regional para la Educación de Adultos en América Latina y el Caribe.

MENDOZA, Mario, 2018. Una fuerza civil. En: GUTIÉRREZ, Gustavo, Cambiando balas por libros: experiencias de lectura en el Ghetto. Colombia: Corporación Biblioghetto. p. 6-9.

MORALES CAMPOS, Estela (Coord.), 2006. Infodiversidad y cibercultura: globalización e información en América Latina. Buenos Aires : Alfagrama.

PORTILLA-LEÓN, Miguel, 2009. La filosofía náhuatl. En: Mendieta, E. y Bohórquez, C. El pensamiento filosófico latinoamericano, del Caribe y "latino" (1300-2000) : historia, corrientes, temas y filósofos. México : Siglo XXI: Centro de Cooperación Regional para la Educación de Adultos en América Latina y el Caribe.

PUEBLOS INDÍGENAS DE OAXACA (2011). Foro Estatal de los pueblos indígenas de Oaxaca. [Acceso 21 de noviembre de 2021] Disponible en http://www.sai.oaxaca.gob.mx/wp-content/uploads/2016/og/foroguelatao.pdf

RESTREPO-FERNÁNDEZ, María Camila. (2020). Alfabetización y lectura: prácticas socioculturales para la inclusión social desde la biblioteca pública. México : UNAM, Posgrado en Bibliotecología y Estudios de la Información. [Acceso 21 de noviembre de 2021] Disponible en http://ru.atheneadigital.filos.unam.mx/jspui/handle/FFYL_UNAM/2144

RIVERA CUSICANQUI, Silvia, 2010. Ch'ixinakax utxiwa : una reflexión sobre prácticas y discursos descolonizadores, Buenos Aires, Retazos-Tinta Limón

SHERA, Jesse, 1976. The library and society. Introduction to library science: basic elements of library service. Littleton, Colorado: Libraries Unlimited.

SHERA, Jesse, 1990. Fundamentos de la educación bibliotecológica [Traducido al español de The Foundations of Education for Librarianship por Sánchez Macgregor, P.]. México: UNAM.

SALAS ASTRAÍN, Ricardo, 2009. La filosofía Mapuche. En: MENDIETA, Eduardo; BOHÓRQUEZ, Carmen. El pensamiento filosófico latinoamericano, del Caribe y "latino" (1300-2000) : historia, corrientes, temas y filósofos. México : Siglo XXI: Centro de Cooperación Regional para la Educación de Adultos en América Latina y el Caribe. p. 4146

Liinc em Revista, Rio de Janeiro, v. 17, n. 2, e5727, nov. 2021. 
VALLEJO, Irene, 2020. El infinito en un junco: la invención de los libros en el mundo antiguo. España : Titivillus. 